\title{
The Sanctuary BVMA in Pescara: AR Fruition of the Pre-Conciliar Layout
}

\author{
Alessandro Luigini \\ Stefano Brusaporci \\ Alessandro Basso \\ Pamela Maiezza
}

Abstract

The project presented here is addressed to the documentation, the investigation of architectural values and their valorization through an application of Augmented Reality technologies enhanced by an Al based tracking application of the Sanctuary Basilica Madonna dei Sette Dolori (BVMA: Beata Vergine Maria Addolorata) in Pescara. The workflow foresees the use of the numerous images taken for the phases of photogrammetric acquisition of the artefact and images taken from the visualizations of the cloud of laser scanner points in order to carry out the "education" phase of the Al software (so that the program can store the greatest number of images for a self interpretative reconstruction of the geometries).The Al data will then be used as a tracking structure for the AR overlay of the digital model on real space, all through a webXR application usable from any device (HMD, desktop or mobile).

Keywords

segmentation, virtual heritage, machine learning for heritage, augmented reality for heritage, deep learning.
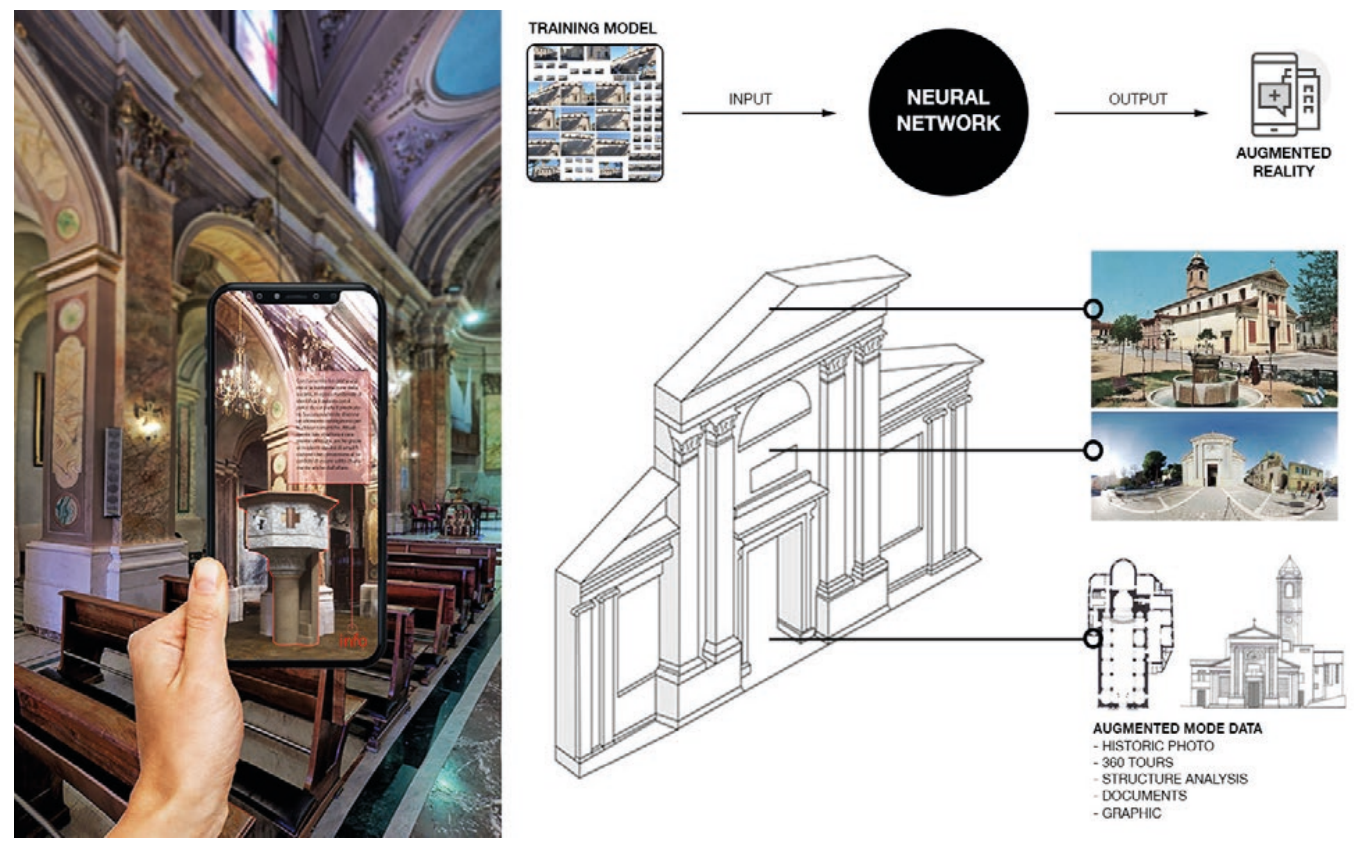


\section{Introduction}

The project is to be included in the context of cultural heritage enhancement practices, through the use of digital technologies of three-dimensional modelling, Augmented Reality and Artificial Intelligence. The aim of the project is the development of an AR navigation device that allows the interactive visualisation of the pre-conciliar configuration of the church in real time. The contribution of digitisation in enhancing heritage and supporting our awareness of our history is significant (Cameron, Kenderine 2007; Pavlidis et al. 2007; Pescarin 20 I6; Luigini 20 I9), and AR technologies add the plus-value of a natural fruition, compared to what happens with VR.

The Sacrosanctum Concilium of 4 December 1963, drafted within the Second Vatican Council held between 1962 and 1965, introduced important innovations concerning the Catholic liturgy and liturgical space that started an important season of adaptation of the existing churches, especially in the area of the presbytery.

The XIX National Eucharistic Congress held in Pescara in 1977 was the opportunity for the upgrading of the Church of BVMA, and the main interventions were: the removal of the balustrade that bounded the presbytery, the replacement of the altar with a frontal altar and the removal of the ambo at the height of the first span, with the consequent placement of the current ambo on the presbytery. The artistic value of the new artefacts does not coincide with those replaced, and so the project to make the early twentieth-century configuration usable again is motivated by the need to restore an architecturally significant configuration to the church.

The church, built in several stages from the second half of the seventeenth century until the mid-nineteenth century with the construction of the bell tower, is in the shape of a Latin cross with three naves covered by elliptical low domes, and a tripartite façade with a pediment in the centre supported by two pairs of pilasters and two side wings with another two pairs of pilasters. The digital reconstruction work will concern, in particular, the apse area and the façade, the latter plastered in the mid-twentieth century and recently exposed (fig. I).

Fig. I. Lateral section of the church obtained from the point cloud of the laser scanner survey.

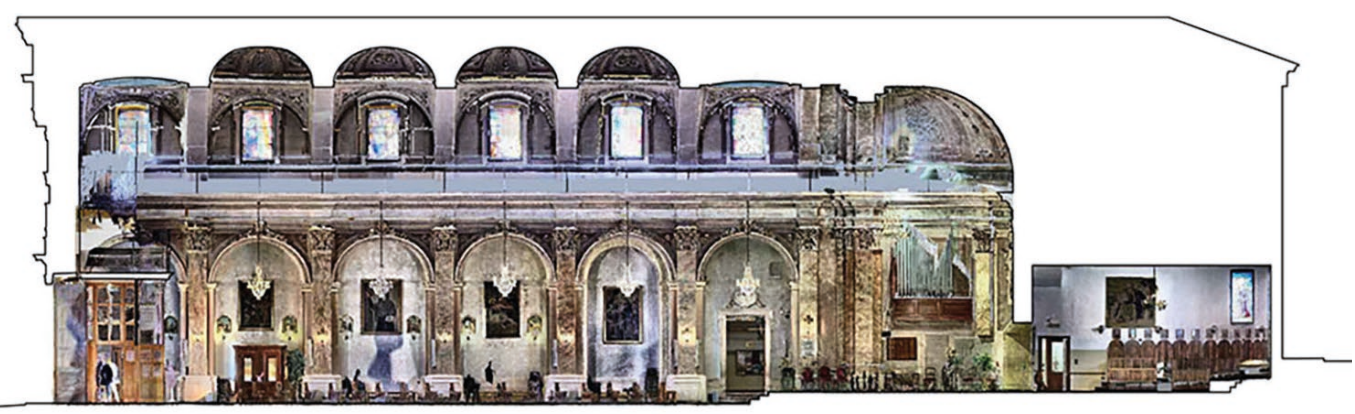

Artificial Logic Construction: State of the Art

Al allows computers to imitate human cognitive processes in order to configure a logic in which 'learning' and 'solving problems' automatically brings considerable benefits in many application areas, where its consistent use can be found in ComputerVision, such as Rendering, Facial Recognition, Video Post Production, but also in 3D survey procedures, laser scanning or photogrammetry, applicable in disciplines such as Geomatics and Architecture: multidisciplinary studies, both theoretical and practical, are progressively opening towards spheres aimed at the analysis and dissemination of Heritage, clearly involving the transversal use of new media, such as AR. New ML technologies and AR dynamics could improve the development of applications that can be exploited in education or Heritage enhancement projects, amplifying space/user interactivity. In the future, a progressive development of smart applications is therefore envisaged, combining the new technologies of $A R, D L$ and Semantic web focusing on the recognition of objects in different conditions, even very complicated ones, retrieving relevant information through semantically linked open data and interactively augmenting this information in real time in a real perceptual environment [Lampropoulos et al. 2020]. 
Fig. 2. Complete workflow of the search process: from document search to AR application.
$A R$ and Al effectively cooperate together, making them the two most promising technologies available to mobile application developers without the need for complex programming steps, since machine learning models use self-generated data from which patterns and correlations are learned, and $A R$, capable of merging physical environments with digital content. Thanks to the integration of the two technologies, the credible superimposition of digital elements on physical objects also makes any element captured, subject to any kind of investigation, questionable through an accurate digital segmentation self-recognised by the computer. This opens up exceptional potential uses by providing new ways of interacting with the physical and digital worlds. The calculations in question refer to the Deep Neural Network, the term 'Deep' expresses the function in which multitudes of data-layers, similar to the human neural system, transform the input data to generate solutions by means of repeated processes of automatic compilation. In short, it is a form of automatic learning that learns to recognise the real world by identifying it through nested hierarchies of visual samples in which each individual conceptual model is defined as the result of other abstractions.

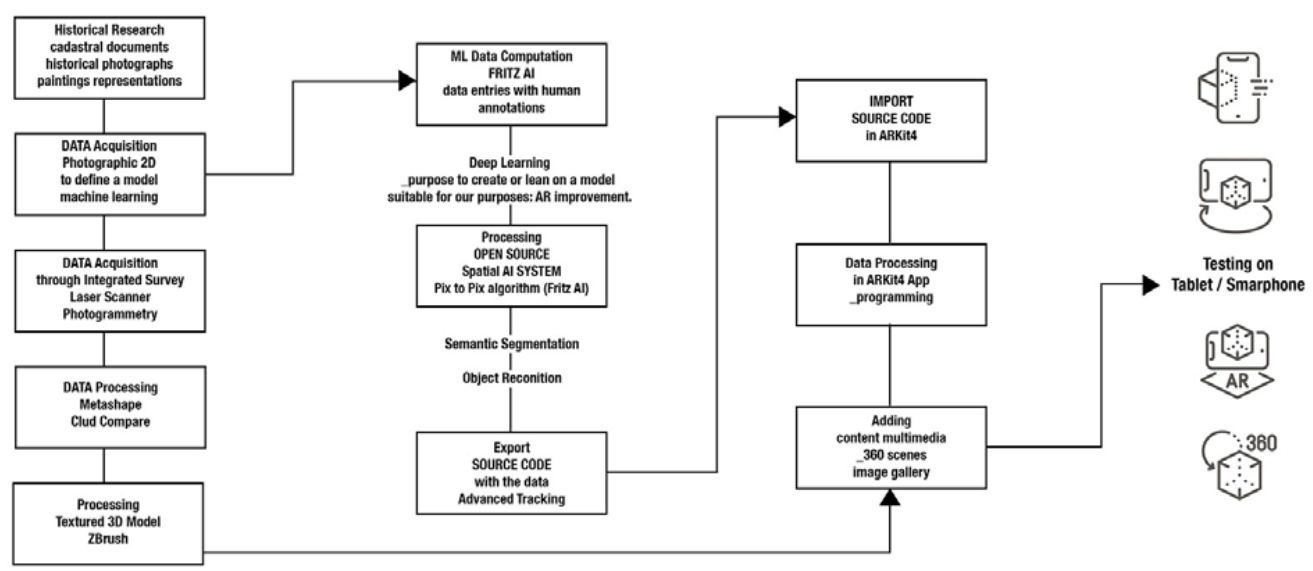

The Application Case Study

This research aims to employ the open source tools available on the web for AR technology with the integration of some algorithms that allow editing of Al-based space3D tracking: a polygonal model of the church of BVMA in Pescara, reconfigured from a previous laser scanner survey according to the latest sculpting modelling techniques, is visually superimposed through an App for smartphones that exploits the most popular AR systems with the support of artificial intelligence according to the preconfigured FRITZ_Al model, to offer a tour that can show, with simplicity and immediacy, the architectural evolution of the church assuming also the previous historical variants (fig. 4). The workflow (fig. 2) can therefore be divided into main phases when the ML procedure employed foresaw the use of numerous images taken from the photogrammetric acquisition and images taken from the post visualisations of the cloud of laser scanner points.

The Al data was then used as a tracking structure for the AR overlay of the digital model on real space, all via mobile devices.

In order to allow in AR visualization a credible correspondence of the model, we opted for a polygonal modeling that exactly traces the point cloud. An HD polygonal model was obtained thanks to the use of the open source software Cloud Compare, through the reconstructive algorithm of the Poisson Surface Reconstruction plug-in, which generated a mesh capable of credibly tracing, based on the parameters entered, the shape that the point cloud assumes from the laser scanner survey starting from its maximum density. In order to obtain a versatile polygonal model suitable for an easy transposition on popular virtual platforms that support AR visualization, it is necessary to optimize the mesh in high resolution deriving from the survey by means of auto-retopology and to generate the UVMap useful to support the textured mappings that replicate the hyper-detailed model, which currently 
Fig. 3. Synthetic diagram of the functioning of GANs, used for tracking, and $\mathrm{CNNs}$, used for semantic segmentation.

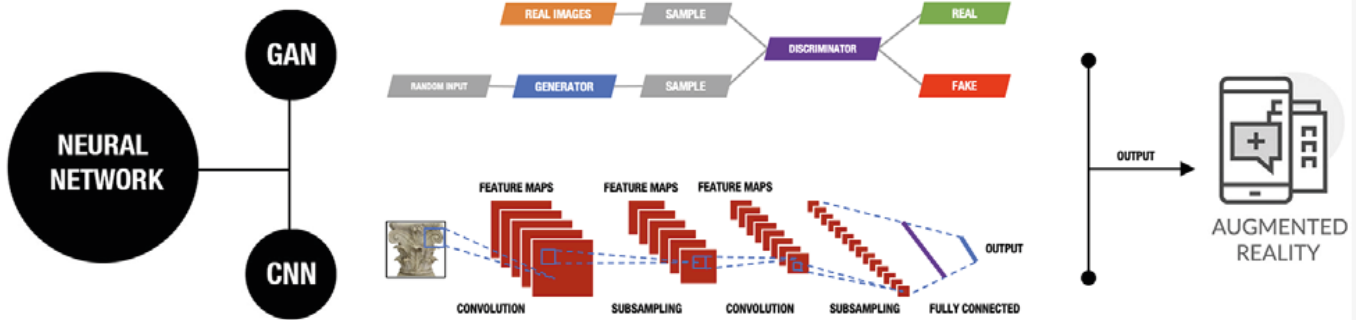

consists of a poly count too high to be managed on mobile real time rendering platforms. The operations are carried out with Zbrush, an artistic sculpting program often used in the workflow for the 3D management of hyper-realistic architectural assets [Trizio et al. 2019]. Machine Learning can currently be considered an indispensable tool for improving AR apps. The case study used the 'FRITZ-AI_ML Platform', an online resource that, by providing Al-based 'training' models, allows developers to use image datasets immediately for production without the need to compile any code. Both Core ML and TensorFlow Lite models are automatically generated, making it easier to develop apps with ARKIT4 and ARCore functionality. Through a univocal interface and a progressive and guided workflow, it is possible to choose the type of model you want to use right from the start, based on the functions supporting the App, including Object Detection, which identifies objects in an image and draws a bounding box around them to make them interrogable, Pose Estimation, which predicts the position of specific key points in an image to perform precise tracking; Image Segmentation, which enables automatic recognition of framed objects using pixels; or Model Labeling, which can recognise people, places and things based on an ML model trained on millions of previously 'labelled' images. For the generation of any model, however, it is necessary to load a Data Set of images functional to the Al training. There are several types of external collections currently in use, such as Oasis Dataset, but for an optimal result the best solution is always to generate, as input of the model to be built, a custom dataset according to the supported mobile ML Frameworks. Thanks to the Dataset Generator function, FRITZ Al offers the opportunity, starting from a few sample figures, to automatically generate a dataset consisting of numerous artificial images with elements ready for intelligent segmentation. In the case study in particular, about twenty sample images taken from the laser scanner survey and deduced from photorealistic renderings carried out specifically by external applications were used. Approximately 500 images were returned, starting from 20 input images, which were then used, in order to configure a $\mathrm{ML} /$ Tensorflow Core Model, in the Al training based on the recognition of the Corinthian capital (applying the Object Reconition Model by means of the input of various images of capitals acquired photographically inside the church) and subsequently on the specific
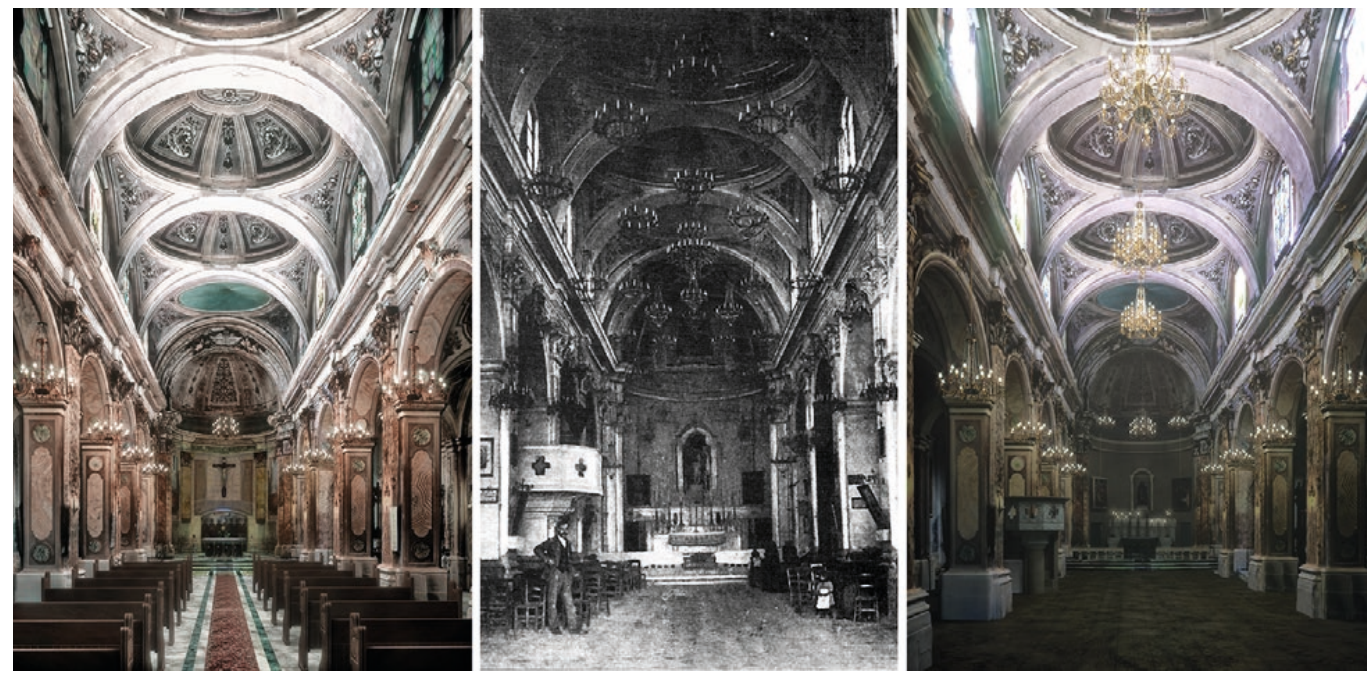
recognition of some key pixels to carry out tracking in the AR environment (applying the Pose Estimation algorithmic Model). In this way it was possible to generate a self-compiled Open Source code that could be subsequently used in the Android framework architectures, in the development of the App currently under completion. The Vuforia system compatible code, thanks to which it is possible to use many of the most common AR functionalities, will allow us in a future phase to link the virtual model to some elements of the frame, in our case decorative elements of extreme recognisability and repetition such as the baroque capitals of the pilasters. The structure will therefore support, especially in the wide framing, where there are more points-target capitals, a solid base to support a stable visualization. As mentioned, it will also be possible to attribute to the elements framed by the cam additional contents, historical data, photo galleries, further model-elements that can be manipulated in 3D. These tools essentially offer the possibility of reducing production times by increasing the quality and consequently the interactivity with the user, a ploy that in short supports engagement policies for the participation of Cultural Heritage.

\section{Conclusion}

Following the digital survey phase, we reconstructed three-dimensionally the interior of the church hall and the exterior, with particular attention to the reconstruction of the pre-conciliar configuration. The following workflow was dedicated to the development of the AR device, able to visualize in a natural way the previous configuration of the church. This device will be used to allow the fruition of the architectural and artistic value of the church, in the most significant configuration of which there is evidence, with the aim of preserving the aesthetic and architectural qualities of the artefact and to spread greater awareness among the public of the values that the local heritage has expressed, also in view of the lack of protection. The awareness-raising programme will include an online communication campaign and activities with local schools, to foster interest in visiting and learning about the heritage.

\section{References}

Asifullah Khan, Anabia Sohail, Umme Zahoora, Aqsa Saeed Qureshi (2020). A Survey of the Recent Architectures of Deep Convolutional Neural Networks. In Artificial Intelligence Review, 54, pp. 5455-55 I 6.

Cameron Fiona, Kenderdine Sofia (2007). Theorizing Digital Cultural Heritage: A Critical Discourse. Cambridge, MA, USA: MIT Press.

Gordon Cooper (2018). Computer Vision for Augmented Reality in Embedded Designs. https://www.edge-ai-vision.com/20 I 8/08/ computer-vision-for-augmented-reality-in-embedded-designs/ online view (0I July 2020).

Lampropoulos Georgios, Keramopoulos Euclid, Diamantaras Konstantinos (2020). Enhancing the functionality of augmented reality using deep learning, semantic web and knowledge graphs: A review. In Visual Informatics, 4, pp. 32-42.

Luigini Alessandro (ed.). (2019). Proceedings of the Ist International and Interdisciplinary Conference on Digital Environments for Education, Arts and Heritage EARTH 2018. Cham: Springer.

Pavlidis George, Koutsoudis Anestis, Arnaoutoglou Fotis, Tsioukas Vassilios, Chamzas Christodoulos (2007). Methods for 3D digitization of Cultural Heritage. In Journal of Cultural Heritage, 8 (I), pp. 93-98.

Pescarin Sofia (2016). Digital Heritage into Practice, In SCIRES-IT, 6, ( I), pp. I-4.

Trizio Ilaria, Brusaporci Stefano, Luigini Alessandro, Ruggieri Andrea, Basso Alessandro, Maiezza Pamela, Tata Alessandra, Giannangeli Alessandro (2019). Experiencing the Inaccessible. A Framework for Virtual Interpretation and Visualization of Remote, Risky or Restricted Access Heritage Places. In ISPRS, 42, pp. I 17 I - 1 I 78.

\footnotetext{
Authors

Alessandro Luigini, Faculty of Education, Free University of Bozen, alessandro.luigini@unibz.it

Stefano Brusaporci, Dept of Civil, Construction-Architectural and Environmental Engineering, University of L'Aquila, stefano.brusaporci@univaq.it Alessandro Basso, Faculty of Education, Free University of Bozen, alessandro.basso@unibz.it

Pamela Maiezza, Faculty of Education, Free University of Bozen, pamela.maiezza@univaq.it 
